\title{
The System of One Dimensional Balls in an External Field. II
}

\author{
Maciej P. Wojtkowski* \\ Department of Mathematics, University of Arizona, Tucson, AZ 85721, USA
}

\begin{abstract}
We modify the system introduced in [W1] so that we can establish the nonvanishing of all Lyapunov exponents easily.
\end{abstract}

\section{Introduction}

In the paper [W1] we have introduced a Hamiltonian system with arbitrary number of degrees of freedom (dimension) for which we can establish the nonvanishing of at least one Lyapunov exponent almost everywhere. It is a system of $n$ particles in a line which fall down with constant acceleration towards a hard floor and collide elastically with each other. This system should be compared with Sinai's gas of hard spheres, for which also the nonvanishing of only few exponents was established in the general case of arbitrary number of spheres ([C-S], [W2]). Although there is little doubt that the system has all exponents different from zero one encounters serious technical difficulties; see the paper about three balls by Krámli, Simányi, and Szász, [K-S-S]. What one needs to prove to get nonvanishing of all exponents is first that every ball is connected by a chain of collisions with every other ball and secondly that certain conspiracies (too technical to formulate here) can occur only on orbits of total measure zero. For our system the former is taken care of automatically (all collisions that can occur do occur on all orbits) but we still cannot prove the latter. In the present paper we modify the potential of the external field from $V(q)=q$ to $V(q)$ such that $f(q)=V^{\prime}(q)>0$ and $f^{\prime}(q)<0$. These requirements allow in particular for the standard gravitational potential $V(q)=-1 / q$. In such a system nonvanishing of all Lyapunov exponents can be established fairly easily under the usual assumption that the masses of the particles decrease as we go up.

\section{Description of the System}

We consider a system of $n$ point particles in the half line $q \geqq 0$ with masses $m_{1}, \ldots, m_{n}$. They collide elastically with each other and the bottom particle collides

\footnotetext{
* Supported in part by the NSF Grant DMS-8807077 and the Sloan Foundation
} 
with the floor $q=0$. They are all under the influence of an external potential field with the potential $V(q)$ such that $f(q)=V^{\prime}(q)>0$ (i.e., the particles fall down) and $f^{\prime}(q)<0$ (i.e., the acceleration decreases with the distance to the floor). Hence between collisions the motion of the particles is governed by the Hamiltonian

$$
H=\sum_{i=1}^{n}\left(\frac{p_{i}^{2}}{2 m_{i}}+m_{i} V\left(\dot{q}_{i}\right)\right)
$$

where $q_{i}$ are the positions and $p_{i}=m_{i} v_{i}$ the momenta of the particles, $i=1, \ldots, n$. At the time of a collision of $i^{\text {th }}$ and $i+1$ particles there is an instantaneous change of momenta

$$
\begin{gathered}
p_{i}^{+}=\gamma_{i} p_{i}^{-}+\left(1+\gamma_{i}\right) p_{i+1}^{-}, \\
p_{i+1}^{+}=\left(1-\gamma_{i}\right) p_{i}^{-}-\gamma_{i} p_{i+1}^{-},
\end{gathered}
$$

where $\gamma_{i}=\frac{m_{i}-m_{i+1}}{m_{i}+m_{i+1}}$, the superscript ${ }^{-}$refers to momenta before the collision and the superscript ${ }^{+}$to momenta after the collision. At the collision of the bottom particle with the floor

$$
p_{1}^{+}=-p_{1}^{-} .
$$

In particular in a collision of two particles the top particle increases its momentum while the bottom particle decreases its momentum by the same amount $\Delta_{i} \mathrm{p}$,

$$
p_{i}^{+}=p_{i}^{-}-\Delta_{i} p, \quad p_{i+1}^{+}=p_{i+1}^{-}+\Delta_{i} p,
$$

where

$$
\Delta_{i} p=\frac{2 m_{i} m_{i+1}}{m_{i}+m_{i+1}}\left(v_{i}^{-}-v_{i+1}^{-}\right)>0 .
$$

In a collision of the bottom particle with the floor its momentum is increased by $\Delta_{0} \mathrm{p}$

$$
p_{1}^{+}=p_{1}^{-}+\Delta_{0} p
$$

where

$$
\Delta_{0} p=-2 p_{1}^{-}>0 .
$$

Our system is a hamiltonian flow with collisions as defined in [W1, Sect. 1]. Indeed we consider the hamiltonian system

$$
\dot{q}_{i}=\frac{p_{i}}{m_{i}}, \quad \dot{p}_{i}=-m_{i} f\left(q_{i}\right),
$$

$i=1, \ldots, n$. We fix the value of the Hamiltonian $H=H_{0}$ and we let $\varphi^{t}: N \rightarrow N$ be the flow defined by (3) on the submanifold $N=\left\{(q, p) \mid H(q, p)=H_{0}\right\} . \varphi^{t}$ preserves the Liouville measure $v$. Further we let $M \subset N$,

$$
M=\left\{(q, p) \in N \mid 0<q_{1}<\ldots<q_{n}\right\} .
$$


The boundary $\partial M$ of $M$ is piecewise smooth and on an open dense subset $\partial M_{r}$ $C \partial M$ the boundary is smooth and the flow $\varphi^{t}$ is transversal to it (regular part of the boundary). The connected components of $\partial M_{r}$ are $\partial M_{i}^{ \pm}, i=0,1, \ldots, n-1$, where

$$
\partial M_{0}^{ \pm}=\left\{(q, p) \in N \mid 0=q_{1}<q_{2}<\ldots<q_{n}, \pm p_{1}>0\right\}
$$

and

$$
\partial M_{i}^{ \pm}=\left\{(q, p) \in N \mid 0<q_{1}<\ldots<q_{i}=q_{i+1}<\ldots<q_{n}, \pm\left(v_{i}-v_{i+1}\right)<0\right\},
$$

$i=1, \ldots, n-1$. The flow $\varphi^{t}$ enters $M$ in $\partial M^{+}$and leaves it in $\partial M^{-}$. The collision rules (1), (2) define the collision map $\Phi: \partial M^{-} \rightarrow \partial M^{+}$. The collision map is symplectic with respect to the canonical symplectic structure on $\partial M_{r}$. Indeed both in the case of the collision with the floor and the collision between two particles $\Phi$ is the restriction of a symplectic linear map of the ambient space $\mathbf{R}^{n} \times \mathbf{R}^{n}$. We have

$$
\left.\Phi\right|_{\partial M_{i}}=\left.\mathscr{Y}_{i}\right|_{\partial M_{i}^{-}},
$$

$i=0,1, \ldots, n-1$ and

$$
\mathscr{S}_{0}=\left(\begin{array}{cc}
I_{1} & 0 \\
0 & I_{1}
\end{array}\right)
$$

where $I_{1}=\operatorname{diag}(-1,1, \ldots, 1)$ is the diagonal matrix with all the diagonal elements equal to 1 except for the first one equal to -1 ,

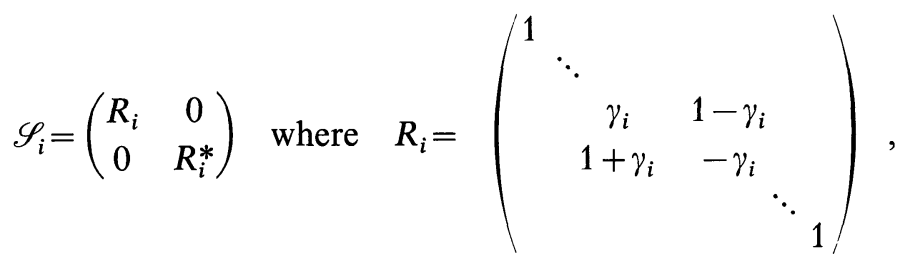

$i=1, \ldots, n-1$. More precisely $R_{i}=\left(r_{k l}\right)_{k, l=1}^{n}$, where $r_{k l}=\delta_{k}^{l}$ (Kronecker's $\left.\delta\right)$ if $k \neq i$, $i+1$ or $l \neq i, i+1$ and $r_{i i}=\gamma_{i}, r_{i, i+1}=1-\gamma_{i}, r_{i+1, i}=1+\gamma_{i}$ and $r_{i+1, i+1}=-\gamma_{i}$.

In this setup we introduce the Hamiltonian flow with collisions $\psi^{t}: M \cup \partial M^{+}$ $\rightarrow M \cup \partial M^{+}$defined by

$$
\psi^{t}(x)=\varphi^{t}(x)
$$

for $0 \leqq t<\tau(x)$, where $\tau(x)$ is the earliest moment of time when $\varphi^{t}(x)$ reaches $\partial M^{-}$ and

$$
\psi^{\tau(x)}=\Phi \varphi^{\tau(x)}(x) .
$$

The flow $\psi^{t}$ preserves the Liouville measure $v$.

We will assume that the total energy $H_{0}$ is smaller than the escape energy of the top mass i.e.,

$$
H_{0}<\sum_{i=1}^{n-1} m_{i} V(0)+m_{n} V(\infty) .
$$

Then the closure of $M$ is compact and the Liouville measure $v$ on it is finite. 
The flow $\psi^{t}$ is not defined or differentiable for all $t$ or $x \in M$. But for $v$ almost all $x D \psi^{t}$ is well defined for all $t$ except for the moments of collision. This allows us to define Lyapunov exponents for the flow. Since $D \psi^{t}$ maps the velocity vector field of the flow into itself we automatically get one zero Lyapunov exponent. It is useful to consider the quotients of the operators $D \psi^{t}$ by the one dimensional subspace spanned by the velocity vector of the flow. We will denote this quotient by $L_{x}^{t}$. What makes the quotient operators useful is that they do not depend on the derivatives of the collision time $\tau(x)\left(D \psi^{t}\right.$ do!).

Theorem. If $f(q)=V^{\prime}(q)>0$ and $f^{\prime}(q)<0$ for all $q, 0 \leqq q \leqq q_{\max }$, where $q_{\max }$ is the highest point the top mass can reach under the energy constraint $H=H_{0}$ (i.e. $H_{0}$ $\left.=\sum_{i=1}^{n-1} m_{i} V(0)+m_{n} V\left(q_{\max }\right)\right)$ and if the masses are decreasing $m_{1}>\ldots>m_{n}$ then the flow $\psi^{t}$ has all but one Lyapunov exponents different from zero.

We will prove the Theorem by applying the $Q$-criterion as formulated in [W1, Sect. 3].

\section{Description of the Derivative}

We introduce linear symplectic coordinates in the tangent spaces to the phase space $\mathbf{R}^{n} \times \mathbf{R}^{n}$ by the formulas

$$
\begin{gathered}
\delta h_{i}=m_{i} f\left(q_{i}\right) \delta q_{i}+\frac{p_{i}}{m_{i}} \delta p_{i} \\
\delta z_{i}=\frac{1}{m_{i} f\left(q_{i}\right)} \delta p_{i}
\end{gathered}
$$

$i=1, \ldots, n$, where $(\delta q, \delta p)$ are natural linear symplectic coordinates in $\mathbf{R}^{n} \times \mathbf{R}^{n}$. In these coordinates the velocity vector field $(3)$ is $(\mathbf{0},-\mathbf{1})$, i.e., $\delta h_{i}=0, \delta z_{i}=-1$, $i=1, \ldots, n$. The tangent space $T_{x} N$ to the submanifold $N=\left\{H=H_{0}\right\}$ becomes

$$
T_{x} N=\left\{(\delta h, \delta z) \mid \delta h_{1}+\ldots+\delta h_{n}=0\right\} .
$$

Let $\mathscr{T}$ denote the quotient space of this tangent subspace by the one dimensional subspace spanned by the velocity vector $(\mathbf{0},-\mathbf{1})$. Note that $(\mathbf{0},-\mathbf{1})$ spans the skeworthogonal complement of $T_{x} N$ and that $\mathscr{T}$ has the canonical symplectic structure.

The linearization of equations (3) is

$$
\delta \dot{q}_{i}=\frac{\delta p_{i}}{m_{i}}, \quad \delta \dot{p}_{i}=-m_{i} f^{\prime}\left(q_{i}\right) \delta q_{i},
$$

$i=1, \ldots, n$. In $(\delta h, \delta z)$ coordinates the equations (6) become

$$
\dot{\delta h_{i}}=0, \quad \dot{\delta} z_{i}=-\frac{f^{\prime}\left(q_{i}\right)}{m_{i} f^{2}\left(q_{i}\right)} \delta h_{i},
$$

$i=1, \ldots, n$. 
We will now express $\mathscr{S}_{i}, i=0, \ldots, n-1$, the linear symplectic extensions of the collision map $\Phi$ in $(\delta h, \delta z)$ coordinates. Since at the moment of a collision of the $i^{\text {th }}$ and $i+1$ particles $q_{i}=q_{i+1}$ and $f\left(q_{i}\right)=f\left(q_{i+1}\right)$, the computations are almost the same as in [W1, Sect. 4], where we had $f(q) \equiv 1$. From (5) we get for $i=1, \ldots, n-1$,

where

$$
\mathscr{S}_{i}=\left(\begin{array}{cc}
R_{i}^{*} & 0 \\
0 & R_{i}
\end{array}\right)\left(\begin{array}{cc}
I & \alpha_{i} K_{i} \\
0 & I
\end{array}\right),
$$

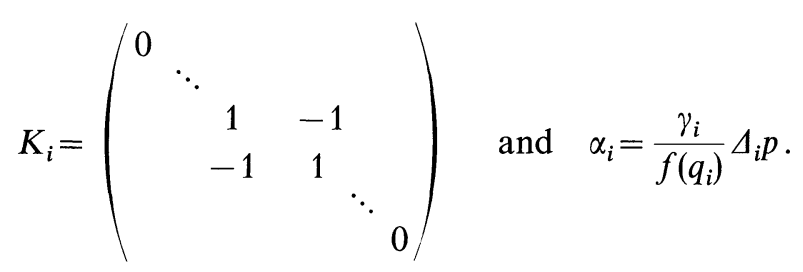

More precisely $K_{i}=\left(k_{l m}\right)_{l, m=1}^{n}$, where $k_{l m}=0$ if $l \neq i, i+1$ or $m \neq i, i+1$ and $k_{i, i}$ $=k_{i+1, i+1}=1, k_{i, i+1}=k_{i+1, i}=-1$. Also using (4) we have

$$
\mathscr{S}_{0}=\left(\begin{array}{cc}
I_{1} & 0 \\
0 & I_{1}
\end{array}\right)\left(\begin{array}{cc}
I & \alpha_{0} F_{1} \\
0 & I
\end{array}\right),
$$

where $F_{1}=\operatorname{diag}(1,0, \ldots, 0)$ is the diagonal matrix with all diagonal elements equal to zero except for the first element equal to one and

$$
\alpha_{0}=f(0) \Delta_{0} p
$$

Now the action of the operator $L_{x}^{t}$ in $\mathscr{T}$ amounts to the effect of (7) interspersed with the application of $\mathscr{S}_{i}, i=1, \ldots, n-1$ or $\mathscr{S}_{0} . \mathscr{S}_{i}, i=1, \ldots, n-1$ factor on $\mathscr{T}$ whereas $\mathscr{S}_{0}$ does not (!) so that the application of $\mathscr{S}_{0}$ should be understood in the following way: immediately before the collision with the floor we project our vector $(\delta h, \delta z)$ onto $T_{x} \partial M_{0}^{-}$along $(\mathbf{0},-\mathbf{1})$ and only then we apply $\mathscr{S}_{0}$.

\section{Proof of the Theorem}

The new element in our approach compared to [W1] is that we consider the operators $L_{x}^{t}: \mathscr{T} \rightarrow \mathscr{T}$ without introducing internal symplectic coordinates in $\mathscr{T}$ or representing $\mathscr{T}$ as an (n-2)-dimensional subspace in $\mathbf{R}^{n} \times \mathbf{R}^{n}$. Instead we work with objects in $\mathbf{R}^{n} \times \mathbf{R}^{n}$ or $T_{x} N$ which factor on $\mathscr{T}$. We begin with introducing a $Q$-form into $\mathscr{T}$.

Definition. The quadratic form $Q$ on the standard linear symplectic space $\mathbf{R}^{n} \times \mathbf{R}^{n}$

$$
Q((x, y))=\sum_{i=1}^{n} x_{i} y_{i}=\langle x, y\rangle, \quad x, y \in \mathbf{R}^{n}
$$

is called the standard $Q$-form. A quadratic form is called a $Q$-form if it is equal to the standard $Q$-form under a linear symplectic change of coordinates.

Lemma. Let $\mathscr{V}$ be a hyperspace in the standard linear symplectic space $\mathbf{R}^{n} \times \mathbf{R}^{n}$ and $\mathscr{L} \subset \mathscr{V}$ the skeworthogonal complement of $\mathscr{V}$ (the characteristic line). If a $Q$-form $F$ on $\mathbf{R}^{n} \times \mathbf{R}^{n}$ factors on $\mathscr{V} / \mathscr{L}$ then $F$ is a $Q$-form on $\mathscr{V} / \mathscr{L}$. 
Proof. Without loss of generality we can assume that

$$
F((x, y))=\langle x, y\rangle, \quad x, y \in \mathbf{R}^{n}
$$

and

$$
\mathscr{V}=\left\{(x, y) \in \mathbf{R}^{n} \times \mathbf{R}^{n} \mid\langle\alpha, x\rangle=\langle\beta, y\rangle\right\} \quad \text { for some } \quad \alpha, \beta \in \mathbf{R}^{n} .
$$

Then $\mathscr{L}$ is spanned by $(\beta, \alpha) \in \mathbf{R}^{n} \times \mathbf{R}^{n}$. Since by assumption $F$ factors on $\mathscr{V} / \mathscr{L}$ we have

$$
F((x, y)+\lambda(\beta, \alpha))=F((x, y)) \text { for every } \quad(x, y) \in \mathscr{V} \quad \text { and } \quad \lambda \in \mathbf{R} .
$$

Hence

and

$$
\langle\alpha, \beta\rangle=0
$$

$$
\langle\alpha, x\rangle+\langle\beta, y\rangle=0 \quad \text { for every }(x, y) \in \mathscr{V} .
$$

We get that either $\alpha$ or $\beta$ is equal to zero so that by an $F$-preserving symplectic change of coordinates we can make

$$
\mathscr{V}=\left\{x_{1}=0\right\} \quad \text { and } \quad \mathscr{L}=\left\{x=0, y_{i}=0, i=2, \ldots, n\right\} .
$$

If we identify $\mathscr{V} / \mathscr{L}$ with $\left\{x_{1}=0, y_{1}=0\right\}$ then the canonical symplectic form in $\mathscr{V} / \mathscr{L}$ becomes $d x_{2} \wedge d y_{2}+\ldots+d x_{n} \wedge d y_{n}$. Hence $F$ on $\mathscr{V} / \mathscr{L}$ becomes the standard $Q$-form.

We introduce now the standard $Q$-form in the $(\delta h, \delta z)$ coordinates

$$
Q((\delta h, \delta z))=\langle\delta h, \delta z\rangle \text {. }
$$

We have

$$
Q((\delta h, \delta z)+\lambda(\mathbf{0},-\mathbf{1}))=Q((\delta h, \delta z))-\lambda\langle\delta h, \mathbf{1}\rangle=Q((\delta h, \delta z))
$$

if $(\delta h, \delta z) \in T_{x} N$, so that $Q$ factors on $\mathscr{T}$ and by the Lemma it is a $Q$-form on $\mathscr{T}$.

We will show that $L_{x}^{t}$ for sufficiently large $t$ (depending on $x \in M$ ) is a strictly $Q$-monotone operator on $\mathscr{T}$. This is equivalent to showing that $D \psi^{t}$ increases the value of the form $Q$ for all vectors in $T_{x} M$ which are not multiples of $(\mathbf{0},-\mathbf{1})$. Between collisions $(\delta h, \delta z)$ evolves according to (7) so that we get

$$
\frac{d}{d t} Q((\delta h, \delta z))=\sum_{i=1}^{n} \frac{-f^{\prime}\left(q_{i}\right)}{m_{i} f^{2}\left(q_{i}\right)}\left(\delta h_{i}\right)^{2} .
$$

Under the assumption $f^{\prime}(q)<0$ the derivative (12) is nonnegative so that between collisions the value of the form $Q$ increases on all vectors $(\delta h, \delta z) \in T_{x} M$ except for vectors of the form $(\mathbf{0}, \delta z)$. By (7) such vectors stay unchanged until a collision of two particles or the collision with the floor. From (8) and (9) we get

$$
Q\left(\mathscr{S}_{i}(\delta h, \delta z)\right)-Q((\delta h, \delta z))=\alpha_{i}\left(\delta z_{i}-\delta z_{i+1}\right)^{2},
$$

$i=1, \ldots, n-1$. Under the assumption that the masses decrease $\left(\gamma_{i}>0\right.$, $i=1, \ldots, n-1)$ and $f(q)>0$ we have $\alpha_{i}>0$ so that the value of the form $Q$ increases in a nondegenerate collision of two particles on every vector $(\delta h, \delta z) \in T_{x} M$ except for vectors with $\delta z_{i}=\delta z_{i+1}$. By (8) and (9) such vectors stay unchanged in the 
collision. Finally by (10) and (11) we get

$$
Q\left(\mathscr{S}_{0}(\delta h, \delta z)\right)-Q((\delta h, \delta z))=\alpha_{0}\left(\delta z_{1}\right)^{2} .
$$

Again under the assumption $f(0)>0$ we have $\alpha_{0}>0$ so that in the collision with the floor the value of the form $Q$ is increased on all vectors except for those with $\delta z_{1}=0$. We cannot claim though that such vectors are unchanged in the collision. On the contrary before the application of $\mathscr{S}_{0}$ we have to project our vector on $T_{x} \partial M_{0}^{-}$ along $(\mathbf{0},-\mathbf{1})$ which typically changes all $\delta z$ coordinates by the same amount but this means that there is no change of the vector in $\mathscr{T}$.

Independently of the initial conditions $x \in M$ every particle will eventually collide with its neighbors (and the bottom particle will collide with the floor). Taking this into account and putting together (12), (13), and (14) we conclude that the only vectors in $T_{x} M$ on which the value of the form $Q$ fails to increase eventually are the vectors with zero $\delta h$ coordinates and equal $\delta z$ coordinates i.e., multiples of $(\mathbf{0},-\mathbf{1})$. We can thus conclude that $L_{x}^{t}$ is strictly $Q$-monotone for sufficiently large $t$ and applying the $Q$-criterion from [W1, Sect. 3] we get the Theorem.

\section{Remarks, etc.}

A. Having established nonvanishing of all Lyapunov exponents for our system we can apply the structural theorems of the Pesin Theory which were worked out for systems with singularities by Katok and Strelcyn [K-S]. Strictly speaking they discussed only the case of discrete time but we can easily represent our system as a suspension of a piecewise smooth map to which the results of [K-S] can be applied. We conclude that our system has at most countably many ergodic components and on each ergodic component the flow is either the Bernoulli flow or a suspension of the Bernoulli system with constant roof function. The question which of the two is the case for our system is wide open. For geodesic or billiard flows this issue is settled once and for all by invoking the underlying contact structure. First of all we have there a family of codimension one invariant subspaces in the tangent bundle and secondly this family is strongly nonintegrable as required by the axioms of a contact structure. This approach was developed in an abstract form by Katok in [K]. In our case we do not know the invariant subspaces. In the case of linear potential $V(q)$ ([W1]) we worked with a family of subspaces which not only fails to be invariant but also is completely integrable (they are tangent spaces of leaves of a nice codimension one foliation of the phase space). In the present paper we consider only the quotient operators $L_{x}^{t}$ and by doing so we altogether avoid looking for invariant subspaces. Naturally then we cannot say anything about the integrability of the family of invariant subspaces (spanned by strongly stable and strongly unstable subspaces).

B. Another question which we did not address is whether our system is ergodic or not. Most probably the methods applied to the gas of hard spheres by Chernov and Sinai [C-S] and Krámli, Simányi, and Szász [K-S-S] are applicable to our system. We can confidently conjecture that our system is ergodic. 
C. Although in the case of a linear potential it does not really matter whether we consider point particles or hard core particles this is not so in the Theorem. The difference surfaces in formula (8) which is not valid any more in the case of hard core particles: at a collision of $i^{\text {th }}$ and $i+1$ particles we have $q_{i+1}-q_{i}=$ diameter of a particle $>0$ and so $f\left(q_{i}\right)>f\left(q_{i+1}\right)$.

D. In the case of a linear potential the exponential growth is the result of interplay between double collisions and collisions with the floor. In the nonlinear case the collisions with the floor are not so important. The only thing we need is that in such a collision the value of the $Q$-form is not decreased. We get the exponential growth from the effect of the evolution (7) between collisions and the double collisions alone.

E. In the case of a potential well of finite depth $(V(\infty)<\infty)$ and sufficiently large value of total energy $H_{0}$ particles may escape to infinity. In such a case one should expect that hyperbolic properties of the system force almost every orbit to infinity. It would be interesting to study the scattering properties of the dynamics, e.g., is it true that for almost all orbits with one $(k)$ particles coming from infinity exactly one (k) particles escape to infinity in the future. What can one say about the scattering map?

F. It is still an open problem whether smooth interaction of particles in a line (pairwise or nearest neighbor) can produce the similar effect of hyperbolic behavior in all of the phase space.

\section{References}

[C-S] Chernov, N.I., Sinai, Ya.G.: Ergodic properties of some systems of 2-dimensional discs and 3-dimensional spheres. Russ. Math. Surv. 42, 181-207 (1987)

[K] Katok, A.: Invariant cone families and stochastic properties of smooth dynamical systems. Preprint (1988)

[K-S] Katok, A., Strelcyn, J.-M. with the collaboration of F. Ledrappier and F. Przytycki: Invariant manifolds, entropy and billiards; smooth maps with singularities. Lecture Notes in Mathematics Vol. 1222. Berlin, Heidelberg, New York: Springer 1986

[K-S-S] Krámli, A., Simányi, N., Szász, D.: Three billard balls on the $v$-dimensional torus is a $K$-flow. Preprint (1988)

[W1] Wojtkowski, M.P.: A system of one dimensional balls with gravity. Commun. Math. Phys. 126, 507-533 (1990)

[W2] Wojtkowski, M.P.: Measure theoretic entropy of the system of hard spheres. Erg. Th. Dyn. Syst. 8, 133-153 (1988)

Communicated by T. Spencer

Received August 1, 1989 